\title{
Modelling of Hot Acoustic Phonon Propagation in Two Dimensional Layers
}

\author{
N. A. BANNOV, V. V. MITIN and F. T. VASKO* \\ Department of Electrical and Computer Engineering \\ Wayne State University, Detroit, MI 48202
}

\begin{abstract}
The transport of confined acoustic phonons in a free-standing quantum well has been studied by solving the quantum kinetic equation for phonons. The phonon decay rate has been numerically calculated for GaAs free-standing quantum well. Phonon interaction with electrons through the deformation potential makes the major contribution to the acoustic phonon decay.
\end{abstract}

Keywords: free-standing structures, confined acoustic phonons, phonon transport

\section{INTRODUCTION}

At present there is a considerable interest toward new type of nanostructures: free-standing quantum wells (FSQWs) and free-standing quantum wires (FSQWIs). An important peculiarity of free-standing structures is the quantization of acoustic phonons. The acoustic phonon quantization has been observed both in optical and electrical experiments [1,2]. The acoustic phonon modes in free-standing structures and their interaction with electrons have been studied in a number of papers (see e.g. Refs. [3-5] and references therein). Due to complicated spectrum of acoustic phonons, their propagation in FSQWs and FSQWIs differs significantly from propagation of bulk acoustic phonons. This is important for heat pulses propagation in such structures and for heat removal from free-standing structures.

In this report we present results of our investigation of the hot acoustic phonon propagation in FSQWs. We have derived the kinetic equation for confined acoustic phonons interacting with $2 \mathrm{D}$ electron gas and analyzed it for the case of phonon transport in the above mentioned structures. We have obtained the renormalization of the acoustic phonon frequencies and relaxation rates due to phonon interactions with electrons through the deformation potential.

\section{KINETIC EQUATION FOR CONFINED PHONONS}

There are three different types of acoustic modes in FSQWs: shear waves, dilatational waves and flexural waves (see e.g. Ref. [5] and references therein). Shear waves are similar to transverse waves in bulk material. Dilatational and flexural waves represent hybrids of longitudinal and transverse waves and differ by their symmetry: dilatational waves are symmetric and flexural waves are antisymmetric in respect to midplane of FSQW. We will denote the frequencies of acoustic modes as $\omega_{m}^{(\alpha)}\left(\mathbf{q}_{\|}\right)$where $\alpha$ is the type of

\footnotetext{
* Present address. Institute of Semiconductor Physics, Kiev 252650, Ukraine
} 


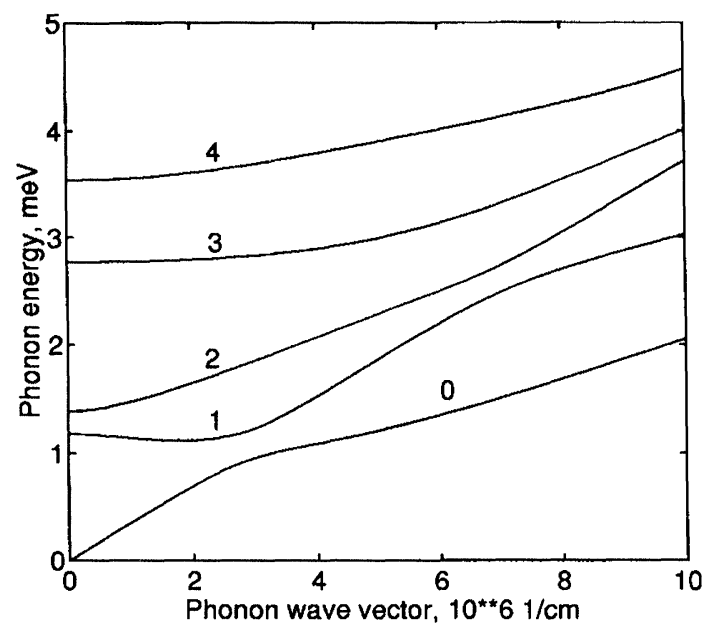

FIGURE 1 Energy dependence for dilatational acoustic modes on the in-plane wave vector. Numbers at curves correspond to the mode numbers. GaAs free-standing quantum well of width $100 \AA$

mode (e.g., dilatational), $m=0,1,2, \ldots$ is the mode number, $\mathbf{q}_{\|}$is a phonon in-plane wave vector. The dispersion relation for five the lowest dilatational acoustic modes calculated for GaAs FSQW $100 \AA$ is shown on Fig. 1.

The Hamiltonian for phonon interactions with electrons through the deformation potential, $H_{e-p}$, is expressed in terms of electron operators, $a_{k}$, and phonon operators, $b_{q}$, in the standard form $H_{e-p}$ $=\Sigma_{k, k^{\prime}, q}\left[w\left(k, k^{\prime}, q\right) a_{k^{\prime}}^{\dagger}, a_{k} b_{q}+\right.$ H.C. $]$, where $q$ denotes the set of phonon variables, $q=\left(m, \alpha, \mathbf{q}_{\|}\right), k$ denotes the set of electron variables, $k=\left(n, \mathbf{k}_{\|}\right)$( $n$ is the electron subband number, $\mathbf{k}_{\|}$is an electron in-plane wave vector), $w\left(k, k^{\prime}, q\right)=\delta_{\mathbf{k}_{\|}^{\prime}, \mathbf{k}_{\|}-\mathbf{q}_{\|}}\left\langle n\left|\Gamma_{m}^{(\alpha)}\right| n^{\prime}\right\rangle$. Here $\left\langle n\left|\Gamma_{m}^{(\alpha)}\right| n^{\prime}\right\rangle$ is the matrix element for the transition, the function $\Gamma_{m}^{(\alpha)}$ is given by the formula

$$
\begin{array}{r}
\Gamma_{m}^{(\alpha)}(z)=\sqrt{\frac{E_{a}^{2}}{2 \mathcal{A} \rho \omega_{m}^{(\alpha)}\left(\mathbf{q}_{\|}\right)}} \\
\left(i \mathbf{q}_{\|} \mathbf{w}_{m}^{(\alpha)}\left(\mathbf{q}_{\|}, z\right)+\frac{\partial \mathbf{w}_{z, m}^{(\alpha)}\left(\mathbf{q}_{\|}, z\right)}{\partial z}\right),
\end{array}
$$

$\mathbf{w}_{m}^{(\alpha)}\left(\mathbf{q}_{\|}, z\right)$ is the normalized vector of relative dispalcement in a phonon mode $(m, \alpha)$, which is given in Ref. [5], axis $\mathrm{z}$ is perpendicular to FSQW, $E_{a}$ is the deformation potential constant, $\mathcal{A}$ is the normalization area; we use such units, that $\hbar=1$.

The kinetic equation for one particle phonon density matrix, $\sigma_{q, q^{\prime}}$, may be derived by averaging the Liou- ville equation over all electron variables and all, but one, phonon variables, and retaining quadratic in $H_{e-p}$ terms (see e.g. Ref. [6]). It has the following form

$$
\begin{gathered}
\frac{\partial \sigma_{q, q^{\prime}}}{\partial t}+i\left(\omega_{q}-\omega_{q^{\prime}}\right) \sigma_{q, q^{\prime}}= \\
-i \sum_{k, k^{\prime}, Q} \frac{w\left(k, k^{\prime}, q^{\prime}\right) w^{*}\left(k, k^{\prime}, Q\right)}{\varepsilon_{k}-\varepsilon_{k^{\prime}}+\omega_{q}-i \lambda} \\
{\left[\left(1-f_{k}\right) f_{k^{\prime}} \delta_{q, Q}+\left(f_{k^{\prime}}-f_{k}\right) \sigma_{q, Q}\right]} \\
+i \sum_{k, k^{\prime}, Q} \frac{w\left(k, k^{\prime}, Q\right) w^{*}\left(k, k^{\prime}, q\right)}{\varepsilon_{k}-\varepsilon_{k^{\prime}}+\omega_{q^{\prime}}+i \lambda} \\
{\left[\left(1-f_{k}\right) f_{k^{\prime}} \delta_{Q, q^{\prime}}+\left(f_{k^{\prime}}-f_{k}\right) \sigma_{Q, q^{\prime}}\right]}
\end{gathered}
$$

It is assumed, that the electron density matrix is diagonal and reduced to the distribution function $f_{k}$. We will also assume, that phonon decay rate and phonon frequency renormalization are relatively small in respect to differences between frequencies of different modes. Then, we may neglect the nondiagonal in respect to discrete variables $m, \alpha$ terms in $\sigma_{q, q^{\prime}}$ and introduce the Wigner function

$$
N_{m}^{(\alpha)}\left(\mathbf{q}_{\|}, \mathbf{r}_{\|}\right)=\sum_{\Delta \mathbf{q}_{\|}} \sigma_{\mathbf{q}_{\|}+\frac{\Delta \mathbf{q}_{\|}}{2} ; \mathbf{q}_{\|}-\frac{\Delta \mathbf{q}_{\|}}{2}} \exp \left(i \Delta \mathbf{q}_{\|} \mathbf{r}_{\|}\right) .
$$

If the spatial variations of the Wigner function are small, Eq. (1) takes the following form

$$
\begin{aligned}
& \frac{\partial N_{m}^{(\alpha)}\left(\mathbf{q}_{\|}, \mathbf{r}_{\|}\right)}{\partial t}+\mathbf{s}_{m}^{(\alpha)}\left(\mathbf{q}_{\|}\right) \frac{\partial N_{m}^{(\alpha)}\left(\mathbf{q}_{\|}, \mathbf{r}_{\|}\right)}{\partial \mathbf{r}_{\|}}= \\
& I_{s p}^{(\alpha)}-v_{m}^{(\alpha)}\left(\mathbf{q}_{\|}\right) N_{m}^{(\alpha)}\left(\mathbf{q}_{\|}, \mathbf{r}_{\|}\right)
\end{aligned}
$$

which is similar to the classical kinetic equation for phonons.

\section{RELAXATION RATES AND RENORMALIZED FREQUENCIES}

The renormalized acoustic phonon velocity in Eq. (2) is equal to

$$
\begin{aligned}
& \mathbf{s}_{m}^{(\alpha)}\left(\mathbf{q}_{\|}\right)=\frac{\partial \omega_{m}^{(\alpha)}\left(\mathbf{q}_{\|}\right)}{\partial \mathbf{q}_{\|}}+ \\
& \left(\frac{\partial \Delta \Omega\left(\mathbf{q}_{1 \|}, \mathbf{q}_{2 \|}\right)}{\partial \mathbf{q}_{1 \|}}-\frac{\partial \Delta \Omega\left(\mathbf{q}_{1 \|}, \mathbf{q}_{2 \|}\right)}{\partial \mathbf{q}_{2 \|}}\right)_{\mathbf{q}_{1 \|}=\mathbf{q}_{2 \|}=\mathbf{q}_{\|}},
\end{aligned}
$$


where correction to phonon frequencies due to electron-phonon interaction is given by the formula $(\mathcal{P}$ stands for the principal value symbol)

$$
\begin{aligned}
& \Delta \Omega\left(\mathbf{q}_{1 \|}, \mathbf{q}_{2 \|}\right)=2 \sum_{n, n^{\prime}, \mathbf{k}_{\|}} \\
& \mathcal{P}\left[\frac{|<n| \Gamma_{m}^{(\alpha)}\left|n^{\prime}>\right|^{2}}{\varepsilon_{n, \mathbf{k}_{\|}}-\varepsilon_{n^{\prime}, \mathbf{k}_{\|}+\mathbf{q}_{1 \|}}+\omega_{m}^{(\alpha)}\left(\mathbf{q}_{2 \|}\right)}\right]\left(f_{n, \mathbf{k}_{\|}}-f_{\left.n^{\prime}, \mathbf{k}_{\|}+\mathbf{q}_{1 \|}\right),}\right.
\end{aligned}
$$

the phonon relaxation rate is determined by the formula

$$
\begin{aligned}
& v_{m}^{(\alpha)}\left(\mathbf{q}_{\|}\right)=4 \pi \sum_{n, n^{\prime}, \mathbf{k}_{\|}} \mid\left\langle n\left|\Gamma_{m}^{(\alpha)}\right| n^{\prime}>\left.\right|^{2}\right. \\
& \delta\left(\varepsilon_{n, \mathbf{k}_{\|}}-\varepsilon_{n^{\prime}, \mathbf{k}_{\|}+\mathbf{q}_{\|}}+\omega_{m}^{(\alpha)}\left(\mathbf{q}_{\|}\right)\right)\left(f_{n, \mathbf{k}_{\|}}-f_{n^{\prime}, \mathbf{k}_{\|}+\mathbf{q}_{\|}}\right),
\end{aligned}
$$

and the rate of the spontaneous phonon emission is given by the formula

$$
\begin{aligned}
& I_{s p}^{(\alpha)}=4 \pi \sum_{n, n^{\prime}, \mathbf{k}_{\|}}|<n| \Gamma_{m}^{(\alpha)}\left|n^{\prime}>\right|^{2} \\
& \quad \delta\left(\varepsilon_{n, \mathbf{k}_{\|}}-\varepsilon_{n^{\prime}, \mathbf{k}_{\|}+\mathbf{q}_{\|}}+\omega_{m}^{(\alpha)}\left(\mathbf{q}_{\|}\right)\right)\left(1-f_{n, \mathbf{k}_{\|}}\right) f_{n^{\prime}, \mathbf{k}_{\|}+\mathbf{q}_{\|}} .
\end{aligned}
$$

We have calculated phonon transport parameters for several the lowest modes (due to selection rules, electrons in the lowest subband of the symmetric quantum well does not interact neither with flexural, nor with shear modes). The results for $v_{m}^{(\alpha)}\left(q_{\|}\right)$are presented on Figs. 2 and results for $I_{s p}^{(\alpha)}$ are shown on Figs. 3. The cut-off at in-plane phonon wave vector $3.1 \times 10^{6} \mathrm{~cm}^{-1}$ on Figs. 2 (a) and 3 (a) are due to high degeneracy of the electron gas for $T=4.2 \mathrm{~K}$; this characteristic wave vector is equal to the doubled Fermi wave vector. The minima in the relaxation rate for zeroth mode at in-plane phonon wave vector $3.2 \times$ $10^{6} \mathrm{~cm}^{-1}$ on Figs. 2 (b) and 3 (b) are associated with a peculiarity of zeroth mode discussed in Ref. [5]. It is interesting to compare the obtained relaxation rates with the acoustic phonon decay rate due to phononphonon interactions. The estimate for spontaneous decay rate of $\mathrm{LO}$ acoustic phonons in bulk GaAs at $\mathrm{T}=4.2 \mathrm{~K}$ is given by the formula [7] : $\mathrm{v}_{s p}=12$
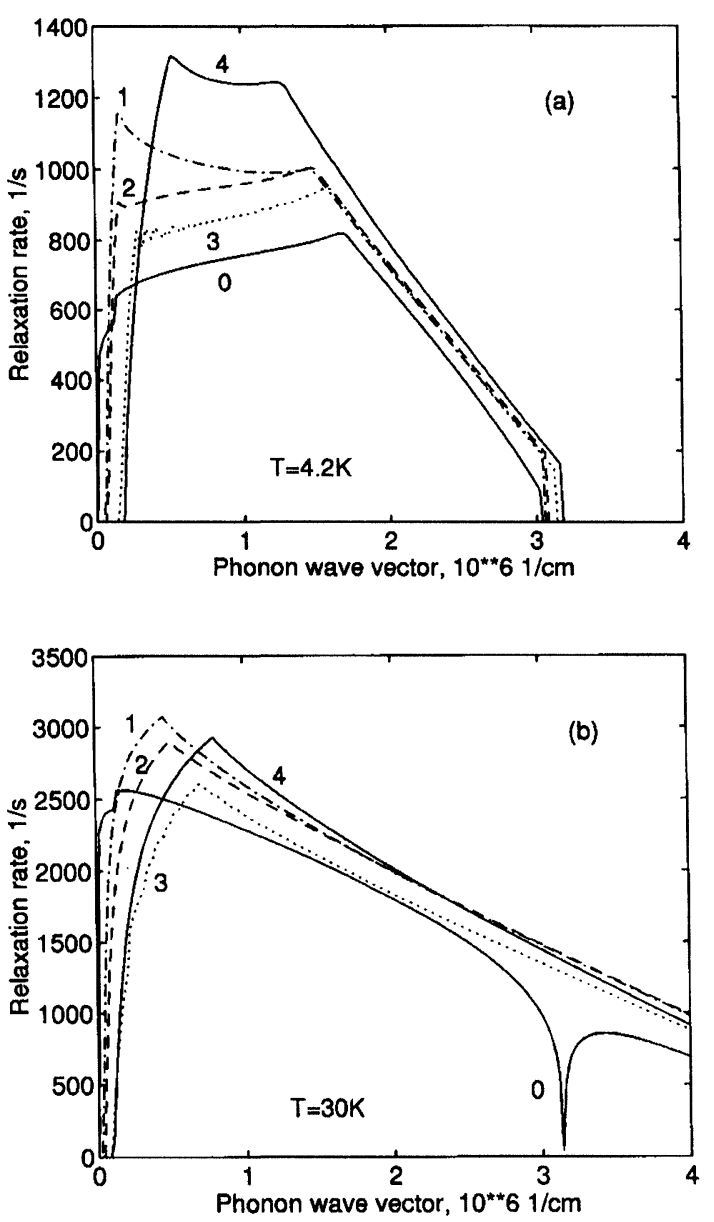

FIGURE 2 Phonon relaxation rates $v_{m}^{(\alpha)}\left(q_{\| 1}\right)$, for five the lowest dilatational phonon modes, $m=0,1,2,3,4$. GaAs FSQW of width $100 \AA$, electron Fermi energy $10 \mathrm{meV}$. The lattice temperature $\mathrm{T}=4.2 \mathrm{~K}(\mathrm{a})$ and $\mathrm{T}=30 \mathrm{~K}(\mathrm{~b})$

$\left(q / 10^{6} \mathrm{~cm}^{-1}\right)^{5} s^{-1}$. Therefore, the phonon relaxation due to the electron scattering dominates in the phonon wave vector range from 0 to $3 \times 10^{6} \mathrm{~cm}^{-1}$. At larger phonon wave vectors the phonon-phonon interaction makes the major contribution to the phonon decay rate.

\section{Acknowledgements}

This work was supported by ARO. 

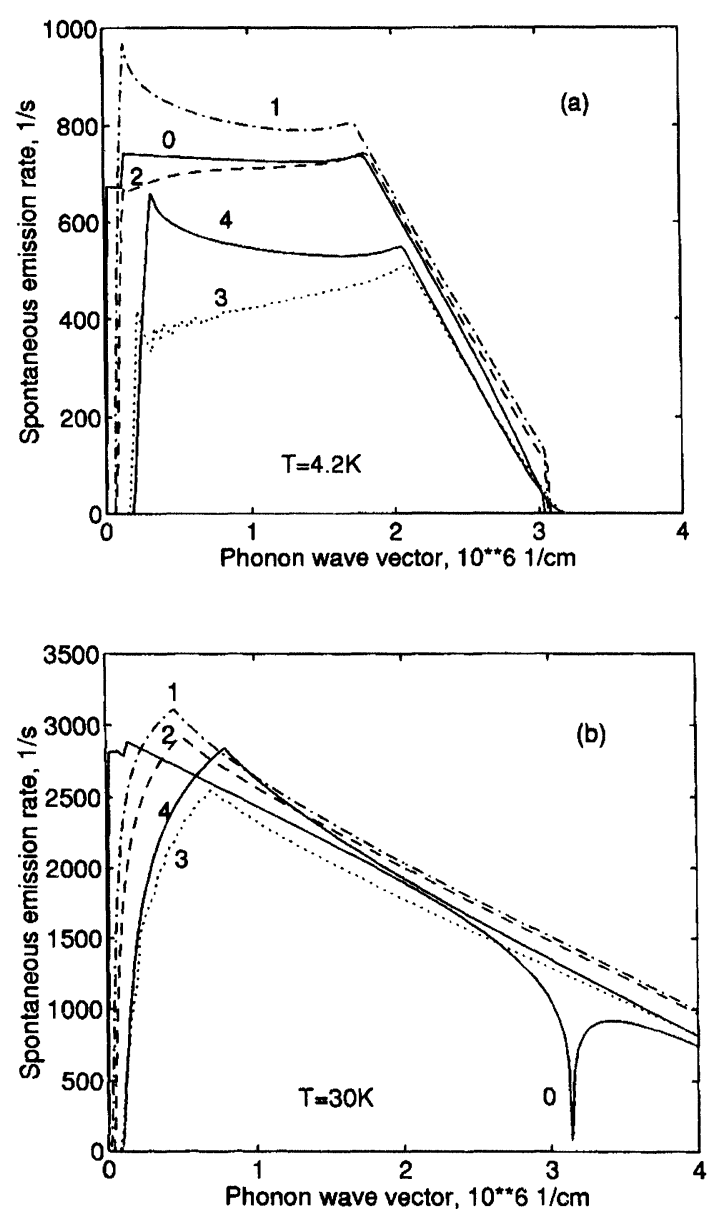

FIGURE 3 Rate of spontaneous phonon emission, $I_{s p}^{(\alpha)}$ for five the lowest dilatational phonon modes, $m=0,1,2,3,4$. GaAs FSQW of width $100 \AA$, electron Fermi energy $10 \mathrm{meV}$. The lattice temperature $\mathrm{T}=4.2 \mathrm{~K}(\mathrm{a})$ and $\mathrm{T}=30 \mathrm{~K}(\mathrm{~b})$

\section{References}

[1] B. Bhadra, M. Grimsditch, I. Schuller, F. Nizzoli, Brillouin scattering from unsupported Al film, Phys. Rev. B 39, 12456 (1989).

[2] J. Seyler and M. N. Wybourne, Acoustic waveguide modes observed in electrically heated metal wires, Phys. Rev. Lett. 69, 1427 (1992).

[3] M. A. Stroscio, and K. W. Kim, Piezoelectric scattering of carriers from confined acoustic modes in cylindrical quantum wires, Phys. Rev. B 48, 1936 (1993).

[4] N. Nishiguchi, Guided acoustic phonons in quantum wires: theory of phonon fiber, Jap. J. Appl. Phys. 33, 2852 (1994).

[5] N. Bannov, V. Aristov, V. Mitin, and M. A. Stroscio, Electron relaxation times due to deformation potential interactions of electrons with confined acoustic phonons in a free-standing quantum well, Phys.Rev.B 51, 9930 (1995).

[6] F. T. Vasko, Emission of acoustic phonons by two-dimensional electrons, Sov. Phys. Sol. State 30, 1207 (1988).

[7] S. Tamura, Spontaneous decay rates of LA phonons in quasiisotropic solids, Phys.Rev.B. 31, 2574 (1985). 

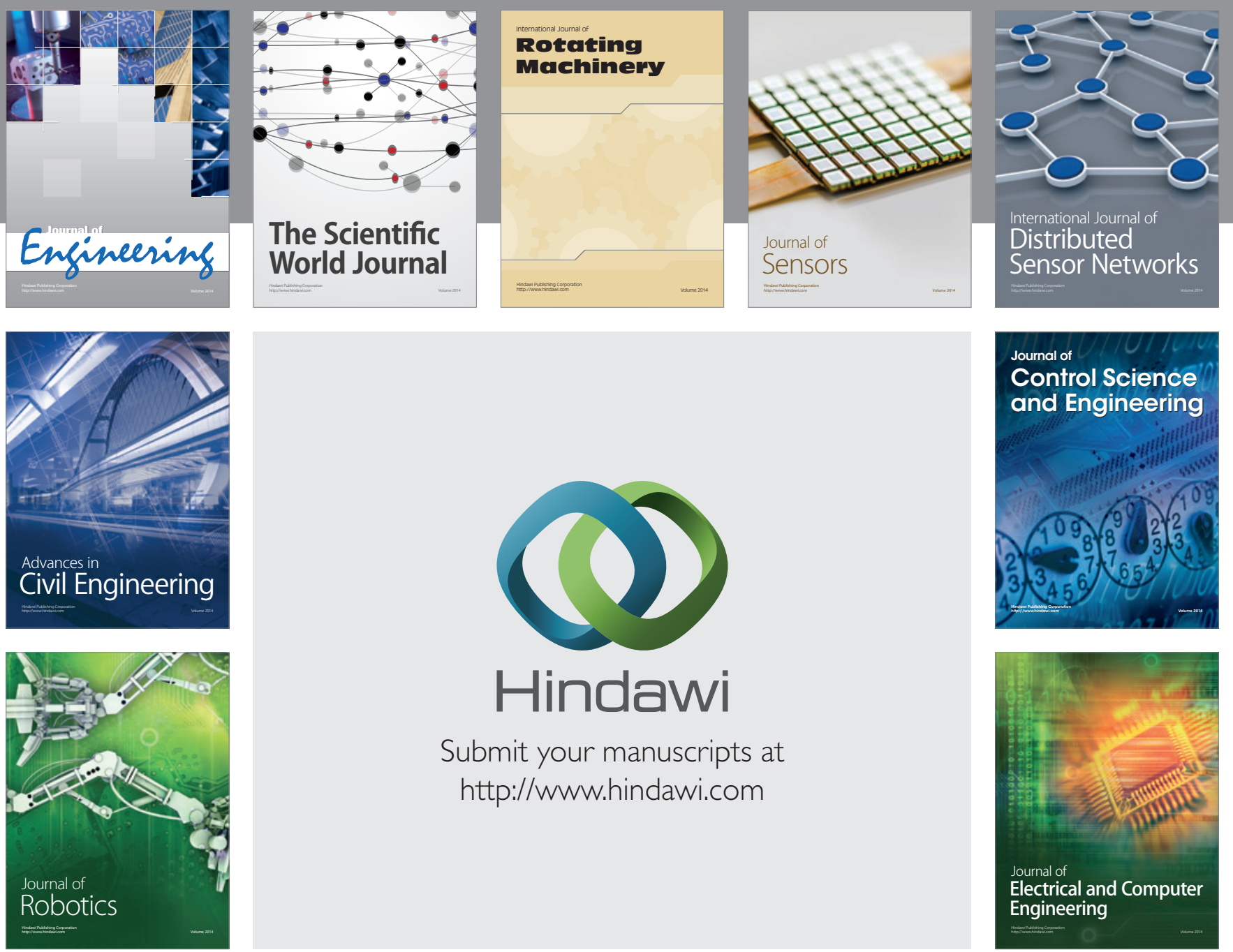

Submit your manuscripts at

http://www.hindawi.com
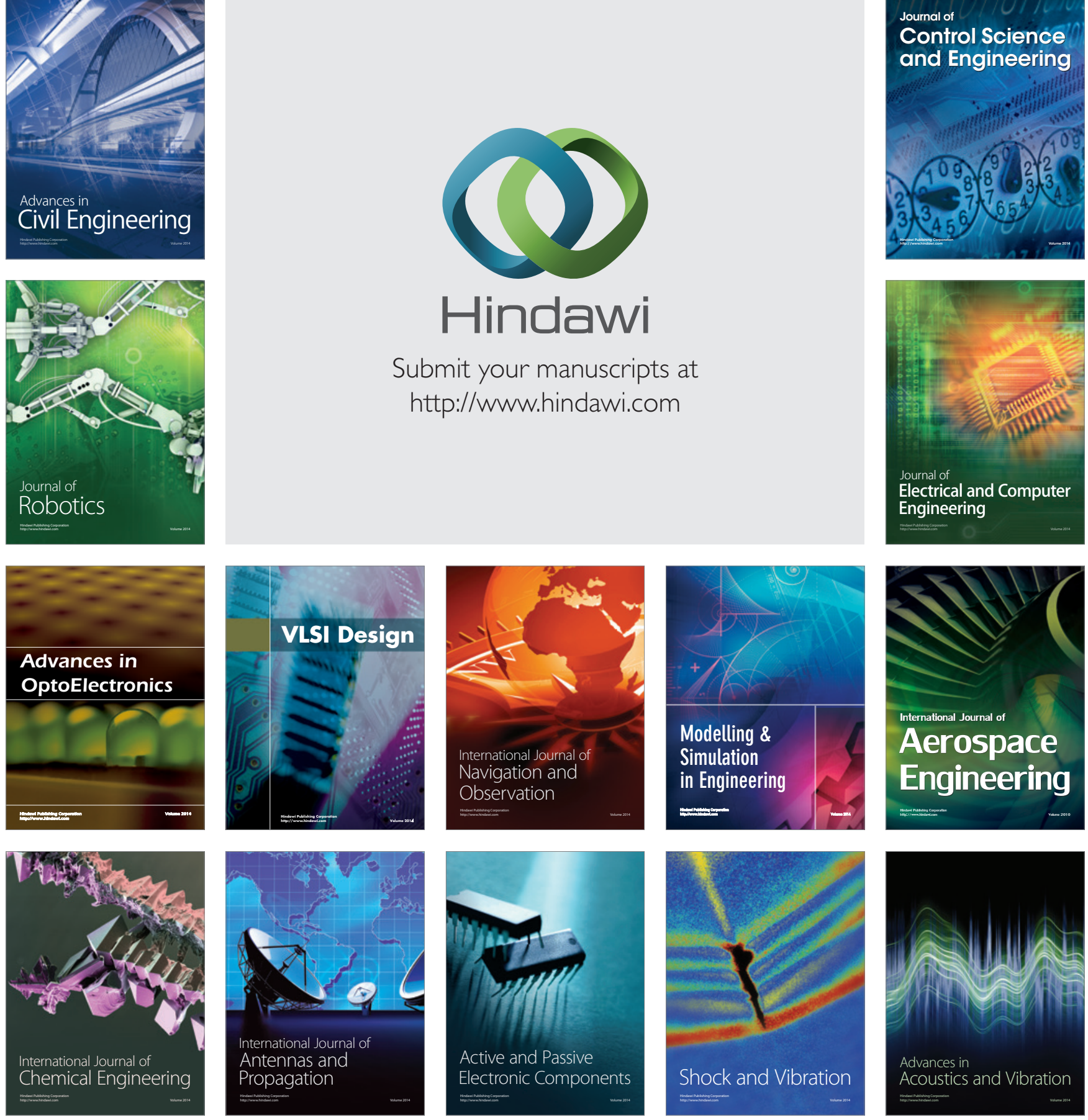